\title{
Programa de media estancia para personas en situación de grave exclusión con necesidades de convalecencia
}

\section{Ernesto Notario}

Albergue Municipal de Elejabarri, Ayuntamiento de Bilbao

<ernesto.notario@ayto.bilbao.net>

\section{Txema Duque Carro}

Sección de Inclusión y Urgencias Sociales, Ayuntamiento de Bilbao

\section{Adela Olascoaga Arrate}

Dirección Territorial de Sanidad y Consumo de Bizkaia, Gobierno Vasco

\section{Karmele Acedo Gil}

Servicios Sociales Integrados, S. Coop.

\begin{abstract}
Etxegabeko egoeran bizi direnek, bestelako biztanle guztiek bezala, osasun-arazoak izaten dituzte, baina gaixotasun eta istripuak izateko arrisku gehiago izan ohi dute. Horrelako arazoak gertatu eta erietxeetan artatzen dituztenean -dela gelan, dela larrialdi-zerbitzuen bidez-, ez dute eriondoko astialdia nonbaiten igarotzerik izaten. Artikulu honetan aurkezten den programak eskaintzen dio etxegabeko egoeran dagoenari premiei egokitzen zaion baliabide bat osasun fisikoaren ondorioz gertatzen diren eriondoko prozesuetan. Gizartebazterketa bizitzen ez duten pertsonen etxebizitzen aurrean ageri den alternatiba egokitu bat da, eta bertan eskuragarri dute ostatua, atsedenaldirako gune bat eta zaintza pertsonalak jasotzea. Baliabide hori Elexabarriko Udal Aterpetxearen baitan dago, eta zentroak dituen baliabide orokorrak erabiltzeaz gain, ekarpena egiten du funtzionalak diren elementu espezifiko batzuek gehituz eriondoko egoeran daude pertsonentzat.
\end{abstract}

\section{HITZ-GAKOAK:}

etxegabeko pertsonak, eriondoko prozesuak, zerbitzuen koordinazioa, koordiontze sozio-sanitarioa, programen ebaluazioa.
Las personas que carecen de domicilio no sólo pueden tener problemas de salud, como la población en general, sino que tienen mayor riesgo de contraer enfermedades o sufrir accidentes. Cuando se presentan estos problemas y son atendidos en centros hospitalarios - sea en planta o en urgencias-, no tienen dónde pasar el tiempo de la convalecencia. El programa que se presenta en este artículo constituye un recurso adaptado a las necesidades de las personas que, careciendo de domicilio, necesitan un periodo de convalecencia por razones de salud física. Es una alternativa necesaria al hogar que disponen las personas no excluidas socialmente, en el que cuentan con alojamiento, lugar de reposo y cuidados personales. Se trata de un dispositivo integrado en el Albergue Municipal de Elejabarri que, utilizando los recursos generales del centro, añade elementos funcionales específicos para las personas en situación de convalecencia.

\section{PALABRAS CLAVE:}

personas sin hogar, procesos de convalecencia, coordinación de servicios, coordinación sociosanitaria, evaluación de programas. 


\section{Marco de funcionamiento}

El 'Programa de media estancia para personas en situación de grave exclusión con necesidades de convalecencia' se puso en marcha el 10 de febrero de 2009. Nació a partir del acuerdo alcanzado entre el Área de Acción Social del Ayuntamiento de Bilbao y la cooperativa de iniciativa social Asociación AurrerantzServicios Sociales Integrados para la prestación de asistencia sanitaria de convalecencia en el Albergue Municipal de Elejabarri. Durante 2009 y 2010, el programa contó con la subvención del Departamento de Asuntos Sociales del Gobierno Vasco. En 2011, fue subvencionado por el Departamento de Sanidad y Consumo del Gobierno Vasco. Y el pasado 1 de febrero de 2012 se firmó un protocolo de colaboración entre la Dirección Territorial de Bizkaia del Departamento de Sanidad y Consumo del Gobierno Vasco, y el Área de Acción Social del Ayuntamiento de Bilbao.

\section{Misión del programa}

El programa procura estancia en lugar adecuado, alimentación, cuidados personales y cuidados sanitarios de baja intensidad a personas con graves problemas de exclusión social y dependencia funcional temporal para las actividades de la vida diaria. Complementariamente, inicia (o da continuidad) a procesos de inserción social de las personas atendidas en la unidad.

\section{Personas destinatarias: requisitos}

El programa se dirige a personas con las siguientes características:

- Mayores de edad de entre 18 y 60 años (excepcionalmente, mayores de esa edad).

- Residentes habituales de Bilbao.

- Indomiciliadas o, excepcionalmente, con viviendas en situación muy precaria y sin convivientes que puedan prestarles cuidados personales.

- Sin trastornos de comportamiento graves que perturben la convivencia colectiva.

- En un estado de salud física que, sin precisar estancia hospitalaria, requiera continuidad de cuidados en domicilio y seguimiento médico.

- Derivados de centro hospitalario tras alta médica emitida bien en servicio de urgencias (por no precisar ingreso), bien en hospitalización (terminado el ingreso).

- Que dispongan de prescripción de tratamientos médicos y, en caso de haber hospitalización previa, indicaciones de continuidad de cuidados de enfermería, aportados por el centro hospitalario.

- Que acepten explícitamente su acogida en el programa mediante la firma y aceptación de las normas de funcionamiento establecidas.

\section{Características generales del recurso}

El programa está integrado física y funcionalmente en el Albergue Municipal de Elejabarri, del Ayuntamiento de Bilbao. Tiene capacidad de atención para nueve plazas.

El ingreso se realiza tras valorar la solicitud en la Comisión Técnica de Valoración y Orientación Sociosaniotaria, compuesta por médico, trabajador social y jefatura de la Sección de Inclusión y Urgencias Sociales. Dispone de una normativa específica de funcionamiento, adaptada a las situaciones de convalecencia.

La estancia máxima es de 45 días, con posibilidad de ampliarla en situaciones excepcionales. Cuando se alcanza ese tiempo y se considera que todavía no se puede dar el alta, el servicio médico que atiende al paciente emite un informe clínico en el que motiva la prolongación de la estancia. Este informe se remite al inspector de la Dirección Territorial de Bizkaia del Departamento de Sanidad y Consumo del Gobierno Vasco, a quien corresponderá dar el visto bueno de la prolongación de la estancia.

\section{Sistema de acceso al programa}

El acceso se realiza a través de solicitud cursada por trabajadores sociales de un centro hospitalario en el formato de informe social y respuesta de aceptación, según el modelo establecido al afecto. De forma extraordinaria, se solicita el ingreso a través de programas de titularidad pública o privada, por el mismo medio. En ambos casos, el informe social irá acompañado de informe médico y la persona usuaria deberá firmar obligatoriamente el documento de aceptación de la normativa del centro y el programa concreto.

\section{Equipo profesional}

La atención médica y de enfermería es cubierta por el centro de atención primaria de Osakidetza de la zona en que se localiza el Albergue Municipal de Elejabarri y por el Servicio de Hospitalización Domiciliaria del Hospital de Basurto. Realizan aportaciones a los usuarios del programa:

- El personal de servicios generales del Albergue Municipal de Elejabarri (personal propio y de servicios contratados por el centro): guardas, vigilantes, personal de cocina, personal de limpieza y de mantenimiento de locales e instalaciones.

- Servicio de Trabajo Social del albergue: trabajadores/as sociales y educadora social.

- Personal específico para el programa: médico (responsable del personal específico) y auxiliar de enfermería (una persona en servicio permanente -24 horas todos los días del año-, en turnos rotativos de mañana, tarde y noche). 
A continuación se describen las funciones de los profesionales del servicio de Trabajo Social del albergue y del personal específico del programa:

- Médico:

- Coordinar el equipo de personal específico (auxiliar de enfermería) en lo relativo a las funciones propias.

- Coordinarse con la Jefatura de Sección de Inclusión y Urgencias Sociales en lo que se refiere al funcionamiento general del programa.

- Coordinarse con la persona responsable del programa en el Albergue Municipal de Elejabarri en lo que respecta a las solicitudes de ingreso y el funcionamiento ordinario del programa.

- Coordinarse con los servicios médicos necesarios.

- Auxiliar de enfermería:

- Proporcionar apoyo para la cobertura de necesidades de cuidado personal, entregar la comida a las personas que no pueden desplazarse al comedor del albergue, acompañar a servicios, facilitar el lavado de ropa personal o movilizar a personas encamadas, entre otras funciones.

- Control de la medicación, según la pauta médica establecida.

- Aplicación y control de cumplimiento de cuidados, según las indicaciones de enfermería.

- Atenciones de enfermería de baja intensidad (circunscritas a las que se encuentran facultadas por su calificación profesional).

- Coordinarse con el/la médico y el/la enfermera/o del centro de salud de la zona.

- Proporcionar apoyo en la reeducación social de los usuarios en cuanto a adquisición de hábitos de la vida cotidiana y habilidades de autocuidado.

- Coordinar el equipo de Intervención Social del Albergue de Elejabarri para el seguimiento de los procesos, mediante participación en las reuniones de equipo establecidas.

- Trabajador/a social:

- Asumir la valoración de situaciones previamente al acceso de las personas usuarias a la unidad, y su aceptación y acogida.

- Realizar el seguimiento del estado de convalecencia para determinar la salida del programa (alta).

- Valorar las posibles situaciones de excepción que justificarían prolongar la estancia más allá de los 45 días establecidos con carácter general.

- Realizar la intervención social requerida para el inicio o la continuidad de procesos de inserción de las personas usuarias.

- Al término de la estancia, obtener (mediante encuesta) la percepción y grado de satisfacción de la persona usuaria y evaluar su progreso en la inserción.

- Aportar información para realizar la evaluación general del programa, según criterios establecidos.

- Educador/a social:

- Prestar apoyo a las personas usuarias realizando los acompañamientos que se requieran, especialmente los necesarios para la asistencia sanitaria ambulatoria cuando existe atención en consultas externas hospitalarias.

- Realizar el acompañamiento social necesario para cumplir el plan de incorporación social.

\section{Instalaciones, mobiliario y cobertura de servicios hosteleros}

Como se deduce de lo anteriormente expuesto, los locales para el programa, dotados del mobiliario básico, los facilita el Albergue Municipal de Elejabarri. En cuanto al mobiliario especial y las ayudas técnicas, Aurretantz S. Coop. incorpora elementos adicionales requeridos por la especificidad del programa: camas articuladas, una grúa, andadores, sillas de ruedas, muletas, adaptador para aseo en cama, adaptador para aseo en ducha y otros elementos auxiliares.

Los servicios de alimentación, limpieza de habitaciones y locales, material de aseo personal y ropa de cama son aportados por el Albergue Municipal de Elejabarri. En este ámbito de servicios hosteleros, se ha previsto la necesidad de medios adicionales, a fin de cubrir las necesidades específicas de las personas usuarias, como alimentación especial, material especial de aseo personal y limpieza, y otros que pudieran presentarse.

\section{Evaluación del programa}

El funcionamiento del programa se evalúa mediante un sistema de evaluación basado en los siguientes criterios: 
Tabla 1. Sistema de evaluación del programa

\begin{tabular}{|c|c|c|}
\hline Criterio & Indicador & Finalidad \\
\hline Nivel de ocupación & $\begin{array}{l}\text { - Número de estancias ocupadas respecto a las } \\
\text { estancias disponibles. }\end{array}$ & $\begin{array}{l}\text { Ajustar la capacidad del programa en cuanto al } \\
\text { número de plazas. }\end{array}$ \\
\hline Índice de respuesta a solicitudes & $\begin{array}{l}\text { - Suma de la diferencia (en días) entre la fecha de } \\
\text { entrada solicitada y la fecha de entrada efectiva, } \\
\text { dividido por el total de entradas programadas. }\end{array}$ & $\begin{array}{l}\text { Valorar la demora respecto a las solicitudes } \\
\text { programadas. }\end{array}$ \\
\hline Duración de las estancias & $\begin{array}{l}\text { - Número de días más frecuente en las estancias } \\
\text { (moda). } \\
\text { - Número de días de estancia mínima. } \\
\text { - Estancias superiores a } 45 \text { días. } \\
\text { - Número de días de la estancia máxima. }\end{array}$ & $\begin{array}{l}\text { Ajustar el tiempo de estancia máxima establecido } \\
\text { con carácter general. }\end{array}$ \\
\hline Tasa de ocupación del servicio & $\begin{array}{l}\text { - Número de estancias /día dividido entre el total } \\
\text { de camas/día disponibles en el recurso (en \%). }\end{array}$ & Optimizar las plazas existentes en el recurso. \\
\hline $\begin{array}{l}\text { Adaptación de los usuarios al } \\
\text { programa }\end{array}$ & - Tasa de abandonos. & $\begin{array}{l}\text { Valorar la eficacia del programa y, en su caso, } \\
\text { incorporar modificaciones en su normativa de } \\
\text { funcionamiento. }\end{array}$ \\
\hline Satisfacción de los usuarios & $\begin{array}{l}\text { - Resultados de la encuesta a los usuarios al } \\
\text { término de la estancia. }\end{array}$ & $\begin{array}{l}\text { Valorar la eficacia del programa desde el punto de } \\
\text { vista de los usuarios e identificar oportunidades de } \\
\text { mejora. }\end{array}$ \\
\hline $\begin{array}{l}\text { Tasa de salidas por alta médica } \\
\text { o ingreso hospitalario }\end{array}$ & $\begin{array}{l}\text { - Suma de salidas por alta médica y por ingreso } \\
\text { hospitalario, dividido entre el total de salidas } \\
\text { (en \%). }\end{array}$ & $\begin{array}{l}\text { Valorar el proceso sanitario de las personas } \\
\text { atendidas. }\end{array}$ \\
\hline $\begin{array}{l}\text { Avances en los procesos de } \\
\text { inserción social }\end{array}$ & $\begin{array}{l}\text { - Evaluación, según escala sencilla, del progreso en } \\
\text { la inserción social de los usuarios al término de } \\
\text { la estancia. }\end{array}$ & $\begin{array}{l}\text { Valorar la eficacia del programa en los procesos de } \\
\text { inserción de las personas usuarias. }\end{array}$ \\
\hline
\end{tabular}

Fuente: Elaboración propia.

\section{Datos de la atención realizada}

\subsection{Ingresos en el programa}

A lo largo de estos años, han ingresado en el programa un total de 198 personas, de las que el $86 \%$ han sido hombres y el $14 \%$, mujeres.

Tabla 2. Ingresos en el programa (2009-2012*)

\begin{tabular}{l|c|c|c|c|c|c}
\cline { 5 - 7 } & \multirow{2}{*}{$\mathbf{2 0 0 9}$} & \multirow{2}{*}{2010} & \multirow{2}{*}{$\mathbf{2 0 1 1}$} & \multirow{2}{*}{$\mathbf{2 0 1 2}^{*}$} & \multicolumn{2}{|c}{ Total } \\
\cline { 5 - 8 } & & & & & $\mathbf{N}$ & $\%$ \\
\hline Hombres & 39 & 50 & 54 & 27 & 170 & 86 \\
\hline Mujeres & 10 & 6 & 6 & 6 & 28 & 14 \\
\hline Total & 49 & 56 & 60 & 33 & 198 & 100 \\
\hline
\end{tabular}

* Primer semestre.

Fuente: Elaboración propia.

De los ingresos programados, el $80 \%$ se han llevado a cabo en la fecha de entrada solicitada y el $20 \%$ restante se han producido con una media de un día de demora.

\subsection{Procedencia y motivos de las solicitudes}

El dato que a continuación presentamos tiene, sin duda, un doble interés. Por una parte, nos sirve para valorar la situación de las personas en el momento inmediatamente anterior a la necesidad del periodo de convalecencia, según la ubicación en la que se encontraban. Por otra parte, resulta útil a la hora de ver cuáles son los estamentos que detectan las necesidades atendidas. Debemos señalar que la práctica totalidad de las personas derivadas desde centros de noche o centros de incorporación social habían sido atendidas por servicios de urgencias de la red sani- taria (hospitalarios u ambulatorios), pero no fueron derivadas al programa de convalecientes, posiblemente porque la intervención fue sólo sanitaria.

Tabla 3. Procedencia (\%)

\begin{tabular}{|c|c|c|c|}
\hline \multirow{5}{*}{$\begin{array}{l}\text { Centros } \\
\text { hospitalarios }\end{array}$} & Hospital de Basurto & 43 & \multirow{5}{*}{48} \\
\hline & Hospital de la Cruz Roja & 2 & \\
\hline & Hospital de Gorliz & 1 & \\
\hline & Hospital de Galdakao & 1 & \\
\hline & Hospital de Cruces & 1 & \\
\hline \multirow{5}{*}{$\begin{array}{l}\text { Albergues, } \\
\text { centros de } \\
\text { noche, centros } \\
\text { de incorporación } \\
\text { social }\end{array}$} & $\begin{array}{l}\text { Albergue Municipal de } \\
\text { Elejabarri }\end{array}$ & 32 & \multirow{5}{*}{52} \\
\hline & $\begin{array}{l}\text { Albergue Municipal de Baja } \\
\text { Exigencia }\end{array}$ & 8 & \\
\hline & $\begin{array}{l}\text { 'Programa de intervención en } \\
\text { calle con personas sin techo' }\end{array}$ & 5 & \\
\hline & $\begin{array}{l}\text { Servicio Municipal de } \\
\text { Urgencias Sociales }\end{array}$ & 5 & \\
\hline & $\begin{array}{l}\text { Entidades que trabajan con } \\
\text { personas sin hogar }\end{array}$ & 2 & \\
\hline
\end{tabular}

Fuente: Elaboración propia.

A la hora de presentar las patologías atendidas (Gráfico 1), debemos señalar que en la mayoría de los casos se presentan varias concomitantes en un mismo paciente (por ejemplo, pie ulcerado, hipoacusia severa, diabetes tipo 2 e insuficiencia renal crónica), por lo que hemos seleccionado la patología que originó la entrada en el programa. En el apartado de 'Otras', quedan aglutinadas patologías como la poliartritis psoriásica, los accidentes cardiovasculares, la litiasis vesical, la hemiparesia o las infecciones del tracto respiratorio con indicación de aislamiento temporal. Por último, hay que remarcar que, además, el $75 \%$ de los usuarios presentan problemas relacionados con el abuso de alcohol u otro tipo de dependen- 
cias, así como patologías infecciosas relacionadas con el consumo de sustancias psicoestimulantes y el consiguiente deterioro psicofísico.

\subsection{Duración de las estancias e índice de ocupación}

La duración media de las estancias (Gráfico 2) ha sido de 34 noches, produciéndose importantes variaciones entre las distintas patologías atendidas (las de mayor duración son las relacionadas con traumatología). En cuanto al índice medio de ocupación del servicio durante el tiempo de funcionamiento, ha sido del $86,3 \%$.

\subsection{Encuesta de satisfacción sobre la atención recibida}

En la Tabla 4 presentamos los resultados de la encuesta de opinión que se realiza a las personas atendidas en el servicio, una vez obtienen el alta.

\subsection{Características de la población atendida}

La edad media de las mujeres atendidas es de 44,9 años, mientras que la de los hombres es de 42,5 años. Este dato se corresponde con el de hombres y mujeres atendidos en situación de calle o en centros de noche y otros dispositivos de incorporación social.
Tabla 4. Valoración de los resultados de la encuesta de satisfacción de usuarios (\%)

\begin{tabular}{l|c|c|c|c|c}
\hline Elemento & Muy mal & Mal & Correcto & Bien & $\begin{array}{l}\text { Muy } \\
\text { bien }\end{array}$ \\
\hline $\begin{array}{l}\text { Información } \\
\text { recibida al entrar } \\
\text { en el programa } \\
\begin{array}{l}\text { sobre sus normas } \\
\text { y funcionamiento }\end{array}\end{array}$ & - & - & 20 & 40 & 40 \\
\hline $\begin{array}{l}\text { Cuidados } \\
\text { recibidos }\end{array}$ & - & - & 20 & 20 & 60 \\
\hline $\begin{array}{l}\text { Amabilidad en el } \\
\text { trato }\end{array}$ & - & - & 20 & - & 80 \\
\hline $\begin{array}{l}\text { Locales y } \\
\text { mobiliario }\end{array}$ & - & - & - & 60 & 40 \\
\hline $\begin{array}{l}\text { Duración de } \\
\text { la estancia en } \\
\text { relación a la } \\
\text { recuperación y } \\
\text { estado de salud }\end{array}$ & - & - & 20 & 60 & 20 \\
\hline $\begin{array}{l}\text { Programa en } \\
\text { general }\end{array}$ & - & - & - & 40 & 60 \\
\hline
\end{tabular}

Fuente: Elaboración propia.

Destaca la franja de edad entre los 36 y los 55 años, que representa un $57 \%$ de la población atendida en el programa.
Gráfico 1. Patologías que motivan el ingreso en el programa (\%)
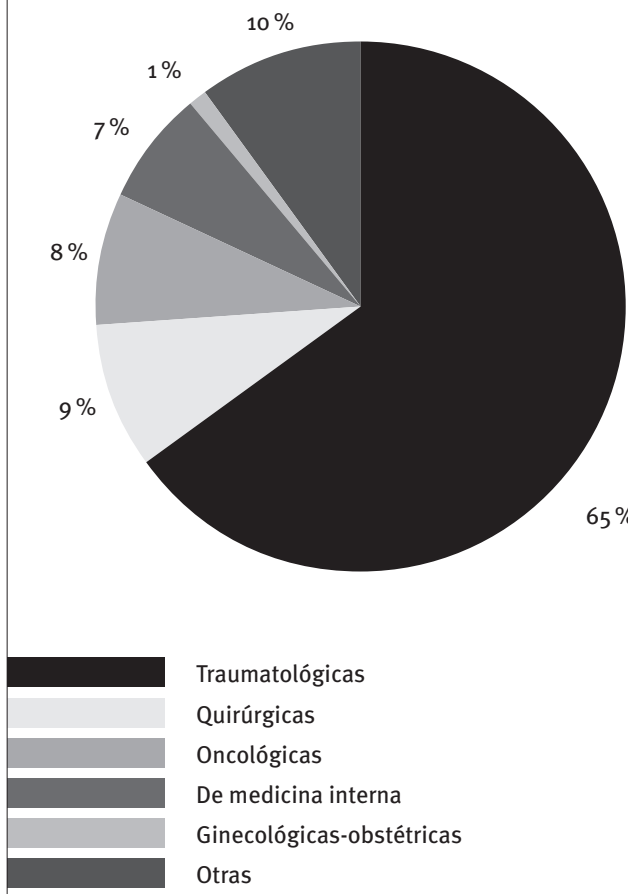

Traumatológicas

Quirúrgicas

Oncológicas

De medicina interna

Ginecológicas-obstétricas

Otras

\section{Gráfico 2. Duración de la estancia, en noches (\%)}
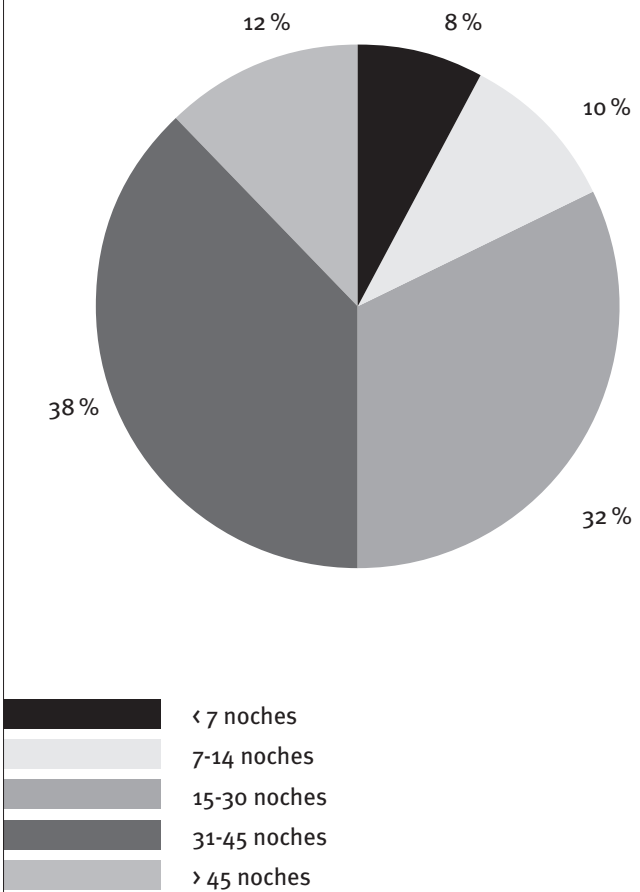

$<7$ noches

7-14 noches

15-30 noches

31-45 noches

$>45$ noches

Fuente: Elaboración propia. 
Tabla 5. Población usuaria, por sexo y edad (\%)

\begin{tabular}{l|c|c|c}
\hline Edad & Hombres & Mujeres & Total \\
\hline$<25$ años & 18 & 0 & 18 \\
\hline $25-35$ años & 12 & 4 & 16 \\
\hline $36-45$ años & 27 & 4 & 31 \\
\hline $46-55$ años & 21 & 5 & 26 \\
\hline$>55$ años & 8 & 1 & 9 \\
\hline Total & 86 & 14 & 100 \\
\hline
\end{tabular}

Fuente: Elaboración propia.

\subsection{País de origen}

El $46 \%$ de las personas atendidas tienen nacionalidad española, mientras que el $54 \%$ son extranjeros, con predominio de personas de los países del norte de África, fundamentalmente Marruecos y, en menor medida, Argelia. Del subgrupo de mujeres, el $90 \%$ son nacionales y tan sólo el $10 \%$ extranjeras. Si cruzamos las variables de edad y país de origen, observamos dos importantes grupos diferenciados:

- Por una parte, un grupo de hombres, menores de 25 años y de nacionalidad marroquí, en situación de infravivienda o con varios meses de estancia en las calles de Bilbao, con patologías traumatológicas producidas a raíz de diversos incidentes, tales como la entrada a lugares de pernocta poco iluminados y de difícil acceso.

- Por otra, un grupo de hombres de nacionalidad española, mayores de 45 años, con un importante historial de adicción al alcohol u a otras sustancias ilegales, que llegan procedentes de centros hospitalarios donde han tenido múltiples ingresos en los últimos años, con infecciones diversas y con dificultades para la adherencia a los tratamientos pautados, por su propio estilo de vida.

Del total de personas atendidas en el programa, el $60 \%$ tienen antecedentes en los servicios sociales municipales de más de 12 meses.

\subsection{Destino al alta}

Además de atender los procesos de convalecencia desde el punto de vista sanitario, las estancias han servido para avanzar en los procesos de incorporación social anteriormente iniciados. Las personas atendidas en el programa que han vuelto a situación itinerario de inserción y que recibín una asistencia puntual, generalmente corta en el tiempo. Las expulsiones realizadas han estado relacionadas con consumos de drogas, y altercados entre usuarios 0 con algunos de los profesionales del programa.
Tabla 6. Destino de los usuarios una vez terminan la estancia en el recurso, según su situación médica (\%)

\begin{tabular}{l|c|l|c}
\hline Con alta médica & $\%$ & Sin alta médica & $\%$ \\
\hline $\begin{array}{l}\text { Continúan proceso de } \\
\text { inserción en alojamiento } \\
\text { temporal }\end{array}$ & 43,2 & $\begin{array}{l}\text { Ingreso } \\
\text { hospitalario }\end{array}$ & 11,4 \\
\hline $\begin{array}{l}\text { Retornan al domicilio familiar } \\
\text { o al alojamiento autónomo }\end{array}$ & 7,9 & $\begin{array}{l}\text { Expulsiones por } \\
\text { incumplimiento de } \\
\text { la normativa del } \\
\text { centro }\end{array}$ & 7,9 \\
\hline Vuelven a situación de calle & 7,9 & Alta voluntaria & 11,4 \\
\hline
\end{tabular}

Fuente: Elaboración propia.

\section{Conclusiones}

El usuario tipo del programa es un varón de nacionalidad española, de entre 35 y 45 años, conocido por los servicios sociales, con una problemática de dependencia a diversas sustancias y con afectación psicofísica derivada de aquélla, con una afectación traumatológica aguda que requiere un periodo de inmovilización y, por consiguiente, de reposo.

El índice medio de ocupación del programa ha sido del $86,3 \%$ y la duración media de las estancias, de 34 noches. Actualmente, el porcentaje de usuarios que, tras el alta, se mantienen en un proceso de inserción -ya sea en albergues o centros de noche, o bien en un alojamiento autónomo- es del $47 \%$. Un $35 \%$ ha obtenido escasos resultados en lo que se refiere a inserción, mientras que el porcentaje restante permanece atendido.

La peculiaridad de nuestro programa de intervención se basa fundamentalmente en tres aspectos:

- La necesidad del periodo de convalecencia.

- El hecho de que vaya dirigido a personas sin hogar.

- El que, dentro de este grupo de población, los pacientes diana sean personas con problemas añadidos relacionados con el abuso de alcohol u otro tipo de dependencias, así como patologías infecciosas relacionadas con el consumo de sustancias psicoestimulantes y el consiguiente deterioro psicofísico.

Se trata de personas que a menudo presentan dificultades para la convivencia normalizada en otro tipo de unidades sanitarias. Tienen tendencia a acumular enseres prácticos desde su punto de vista, pero $\sin$ duda mediatizados por vivir durante periodos prolongados en situaciones de extrema necesidad. Les cuesta acatar la normativa relacionada con hábitos de higiene, $\mathrm{y}$ tienen pocas habilidades relacionales tanto con los profesionales del programa como con otros usuarios. Estas peculiaridades, junto con los consumos de sustancias psicoestimulantes o depre- 
soras del sistema nervioso central, han provocado el número de abandonos y expulsiones señaladas anteriormente.

Asimismo, debemos destacar la importancia de la rapidez en la respuesta a las demandas planteadas. Si el tiempo medio de espera para el ingreso en el

\section{Referencias bibliográficas}

ARARTEKO (2008): Atención sociosanitaria: una aproximación al marco conceptual y a los avances internacionales y autonómicos, serie Informes Extraordinarios, VitoriaGasteiz, Ararteko [<http://www.ararteko.net/ RecursosWeb/DOCUMENTOS/1/2_1357_3.pdf)].

MINISTERIO DE SANIDAD, POLÍTICA SOCIAL E IGUALDAD (2011): Configuración de una red local de atención a personas sin hogar integrada en el Sistema Público de Servicios Sociales. Cien programa ha sido de un día, no se debe a la casualidad, sino a las características del grupo atendido, pues esa urgencia en la intervención está directamente relacionada con la oportunidad de apoyar situaciones de personas con claros rasgos de abandono de sus necesidades personales. argumentos y propuestas, serie Informe, Estudios e Investigación 2011, Madrid, Ministerio de Sanidad, Política Social e Igualdad [rhttp://www.msps.es/gl/novedades/ docs/10oargumentosEspayol.pdf $>$ ].

PAÍS VASCO (2008): “Ley 12/2008, de 5 de diciembre, de Servicios Sociales", Boletín Oficial del País Vasco, 24-12-2008, nํ2 246, págs. 31.84031.924 [<http://www.euskadi.net/bopv2/ datos/2008/12/0807143a.pdf $\rangle$. 